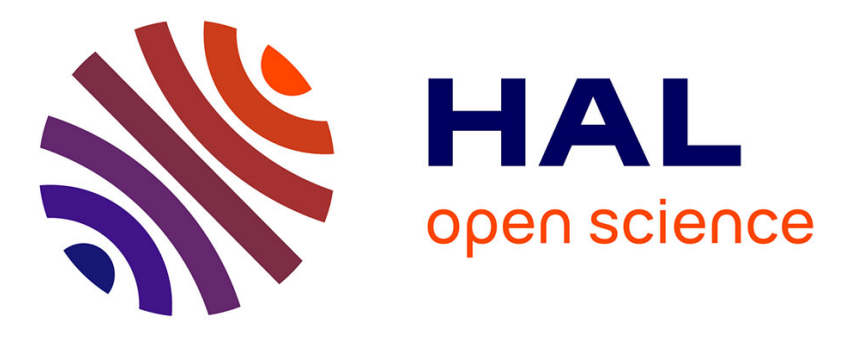

\title{
When do imperfectly competitive firms maximize profits? The lessons from a simple general equilibrium model with shareholders' voting
}

Rim Lahmandi-Ayed, Didier Laussel

\section{- To cite this version:}

Rim Lahmandi-Ayed, Didier Laussel. When do imperfectly competitive firms maximize profits? The lessons from a simple general equilibrium model with shareholders' voting. Journal of Mathematical Economics, 2018, 78, pp.6-12. 10.1016/j.jmateco.2018.06.006 . hal-01991962

\section{HAL Id: hal-01991962 \\ https://hal-amu.archives-ouvertes.fr/hal-01991962}

Submitted on 31 Jan 2022

HAL is a multi-disciplinary open access archive for the deposit and dissemination of scientific research documents, whether they are published or not. The documents may come from teaching and research institutions in France or abroad, or from public or private research centers.
L'archive ouverte pluridisciplinaire HAL, est destinée au dépôt et à la diffusion de documents scientifiques de niveau recherche, publiés ou non, émanant des établissements d'enseignement et de recherche français ou étrangers, des laboratoires publics ou privés.

\section{(이) $\$$}

Distributed under a Creative Commons Attribution - NonCommercial - NoDerivatives 44.0 


\title{
When do imperfectly competitive firms maximize profits? The lessons from a simple general equilibrium model with shareholders' voting
}

\author{
Rim Lahmandi-Ayed ${ }^{\mathrm{a}, *}$, Didier Laussel ${ }^{\mathrm{b}}$ \\ a ESSAI and UR MASE-ESSAI, Université de Carthage, Tunisia \\ ${ }^{\mathrm{b}}$ Aix-Marseille University (Aix-Marseille School of Economics), CNRS EHESS, France
}

\begin{abstract}
We consider a general equilibrium model with vertical preferences, where workers and consumers are differentiated, respectively, by their sensitivity to effort and their intensity of preference for quality. We consider a monopoly of which the shares are owned by a fraction of the general population. The price is determined through a vote among all the shareholders. We identify necessary and sufficient conditions for (i) an absolute (relative) majority to vote for the profit maximizing price; (ii) an absolute (relative) majority to vote for a different price. We argue that the more concentrated the ownership the more likely it is that the firm charges the profit-maximizing price.
\end{abstract}

Keywords: General equilibrium Profit maximization Vertical preferences Majority vote

\section{Introduction}

Though its shareholders may have diverging preferences and may pursue very different interests, it is standard in the economic literature to assume that a firm's unique objective is profit maximization. This assumption is perfectly consistent with the heterogeneity of shareholders' objectives as long as the firm is a price-taker. But it has long been recognized (see for instance Marshall, 1920) that this ceases to be true when one moves to an imperfectly competitive environment. ${ }^{1}$ This has been emphasized among others by Gabszewicz and Vial (1972), Cornwall (1977), Hart (1985) and Bonnano (1990). The issue is that an imperfectly competitive firm, by affecting the prices of inputs and outputs, influences the consumption possibilities of its owners. As put by Hart (1985, page 107): “..the owners of a firm are interested not in monetary profits per se, but rather in what this profit can buy. Given that a monopolistically competitive firm can influence prices, the owners may prefer low monetary profit but favorable prices for consumption goods to high monetary profit and unfavorable prices". In addition, "If owners have different tastes, they will have different trade-offs concerning high monetary profit versus favorable consumption goods prices. That is, each owner will have his own private objective function which he would like the firm

\footnotetext{
* Corresponding author.

E-mail address: rim.lahmandi.ayed@gmail.com (R. Lahmandi-Ayed)

1 Dierker and Grodal (1999) interestingly remembered that "the decision problem a multi-owner firm has to face presented a major motivation for K. Arrow to develop his theory of social choice".
}

to pursue, and the problem then is how to aggregate these into an overall objective function".

Despite these remarkable insights, all general equilibrium models with imperfect competition ${ }^{2}$ (Gabszewicz and Vial, 1972; Nikaido, 1975; Codognato and Gabszewicz, 1991; Gabszewicz and Michel, 1997; Guesnerie and Hart, 1985); among others have chosen to stick to the profit maximization hypothesis. However, as put by Cornwall (1977, pp 56-58) "it is equally clear that it is not enough to say that there are a lot of firms in a real world economy and that therefore the assumption of profit-maximizing behavior gives a good approximation. This is not enough of a justification because it is not clear what or how profit maximization approximates".

Moreover, as emphasized by Dierker and Grodal ${ }^{3}$ (1999, under imperfect competition, profit-maximization may yield very different equilibrium outcomes, according to the "normalization rule" which converts relative prices into absolute prices so that assuming profit-maximization is not enough: one needs in addition to precise in what units profits are to be measured, i.e. what is the numeraire (the normalization rule).

The present note derives, from a very simple general equilibrium model a necessary and sufficient condition which ensures that a monopolistic firm, whose owners' preferences are heterogeneous, chooses the profit-maximizing strategy. This is more precisely a condition which ensures that there is in the firm's board of

\footnotetext{
2 Not to speak of partial equilibrium models.

3 Dierker and Grodal (1999) is among the rare examples of papers which do not stick to the profit maximization hypothesis.
} 
directors an absolute (relative) majority of shareholders who favor maximization of profits, when profits are measured in units of a (composite) consumption good which is neither an input nor an output. ${ }^{4}$ These shareholders rationally expect neither to consume the product nor to work in the firm at their ideal price, i.e. the profitmaximizing one. ${ }^{5}$ If they are an absolute majority, their ideal price will emerge from any voting method inside the board of directors. This condition is stronger than the one which would simply ensure that the profit-maximizing price be a Condorcet winner ${ }^{6}$ (on this concept and related ones, see for instance Persson and Tabellini (2002), chapters 2 and 3) or from a relative majority condition corresponding to a plurality voting system. Despite the simplicity of the model, we shall argue that it yields rather general insights.

We use the small general equilibrium model developed in Kahloul et al. (2017) in the monopoly case with an uniform ownership and an exogenous quality of the product, but supposing here that shareholders determine through voting the firm's price instead of assuming that the monopolist chooses the profitmaximizing price. The economy encompasses a single firm producing a vertically differentiated product using labor as the unique input and a population of workers/consumers/shareholders characterized by two parameters: intensity of preference for product quality and sensitivity to effort. Each individual decides whether to work or not and decides whether to purchase one unit of the product or not, in order to maximize his/her utility given his/her income. When the price is set at some given level, the salary adjusts in order to equalize demand and supply on the labor market. The firm is supposed to be equally possessed by a given fraction of the population.

We identify necessary and sufficient conditions under which (i) there is a majority (absolute or relative) of shareholders who chooses the price which maximizes the firm's profit, (ii) there is a majority (absolute or relative) of shareholders who chooses a price which does not maximize the profit (i.e. the ideal price of shareholders who work in the company and consume its products).

The note is organized as follows. Section 2 provides the model. Section 3 gives the main results. Section 4 concludes.

\section{The model}

Consider an economy with 3 goods: labor as the input, a numeraire and an indivisible "differentiated" good as the unique output ("differentiated" in the sense that it may possibly be of different qualities perceived differently by consumers).

There is a population of workers/consumers/shareholders. Each individual is endowed with an indivisible unit of labor and a given quantity $e$ of a (composite) numeraire $\operatorname{good}^{7}$ and we denote by $\lambda \geq 0$ his/her uniform share in the firm's profit. Each agent $(\alpha, \theta)$ is doubly characterized by

- a "working parameter" $\alpha \in[0, \bar{\alpha}]$ which captures the worker's sensitivity to effort,

- a "consumption parameter" $\theta \in[0, \bar{\theta}]$ which measures the intensity of the consumer's preference for the product's quality.

\footnotetext{
4 We think that this is a more natural way of defining profit-maximization when the firm is a price-setter: profit-maximization corresponds to the maximization of the number of units of the composite good that its pure shareholders can consume.

5 Notice that the shareholders whose ideal price is the profit-maximizing one are a subset of the set of shareholders who choose not to consume nor to work at the profit-maximizing price. This is made clear later on.

6 Notice that the Condorcet winner is not always elected, depending on the voting method.

7 The relative prices of the consumption goods which compose the numeraire are considered by the shareholders as fixed or, at least, are not influenced by the price of the monopoly good.
}

Individuals are uniformly distributed over $[0, \bar{\alpha}] \times[0, \bar{\theta}]$ with a density equal to 1 . Each worker/consumer $(\alpha, \theta)$ chooses sequentially:

- first, whether to remain idle $(\bar{W})$ or to work $(W)$ in the differentiated sector,

- then to compose his/her consumption bundle, in particular whether to consume $(C)$ or not $(\bar{C})$ one unit of the differentiated product.

One firm produces the differentiated product. One unit of the differentiated good requires one unit of labor.

Individuals derive their utility from the consumption of these two goods ${ }^{8}$ as follows:

$V(x, t)=\theta q x+t$,

where

- $x$ is the consumption of the differentiated product of exogenous quality $q$,

- $t$ is the consumption of the numeraire good,

- the consumption bundle $(x, t)$ must belong to the consumption set $\{0,1\} \times \mathbb{R}$.

If the individual chooses to work, he/she receives a salary $\omega$ and must incur a training cost $\alpha q$. If he/she chooses not to work, he/she receives no salary (and does not have to be trained), his/her revenue being limited to the initial endowment in the numeraire and to his/her share in the firm's profit.

We suppose that the individuals are split into two groups: a fraction $\mu \in[0,1]$ are shareholders, with $\lambda=\frac{1}{\mu \bar{\alpha} \bar{\theta}}$, and a fraction $1-\mu$ are non-owners, for whom $\lambda=0$. A decrease in $\mu$ corresponds to a more concentrated ownership. Only shareholders vote to determine the price. Notice that, in order to isolate the influence of the concentration of ownership, we assume that the distributions of the parameters $\alpha$ and $\theta$ are the same in the population of shareholders as they are in the general population of agents.

The firm's price is determined through a vote by all shareholders. The salary adjusts such that the labor's demand and offer equalize, so that

$\omega=\bar{\alpha}\left(q-\frac{p}{\bar{\theta}}\right)$.

The firm's profit is then the following function of its price ${ }^{9}$ :

$\pi=\bar{\alpha}\left[p\left(1+\frac{\bar{\alpha}}{\bar{\theta}}\right)-\bar{\alpha} q\right]\left[\bar{\theta}-\frac{p}{q}\right]$.

Concerning the issue of the vote, we shall consider that the winning price is the winner in a majority vote among the shareholders. We shall analyze in particular the case where an absolute majority is required ${ }^{10}$ and determine the conditions under which the profit maximizing price is selected under this rule. But we shall also analyze the weaker conditions which emerge in the case of a plurality (relative majority) system.

\section{To maximize the profit or not to maximize the profit?}

We prove that only two prices may be chosen by shareholders in a plurality system: the profit maximizing price, the price which is the most preferred by pure shareholders, ${ }^{11}$ and a different price,

\footnotetext{
8 The quality parameter $q$ and the initial endowment $e$ do not play a role in the present work. They are maintained to allow properly further developments.

9 For details on the derivation of the results see Kahloul et al. (2017).

10 Obviously if there is an absolute majority of shareholders who prefer some price, it is a Condorcet winner.

11 By pure shareholders we mean shareholders who do not buy the product nor work in the firm.
} 
namely the price most preferred by shareholders who work in the firm and consume its products.

We identify necessary and sufficient conditions on the model's parameters under which an absolute or a relative majority of shareholders favor the profit maximizing price and necessary and sufficient conditions under which the second price is favored by a majority (absolute or relative) of shareholders. This is the object of Proposition 1.

It is straightforward to calculate the ideal price of a given shareholder, conditional on $(X, Y)$, where $X=C, \bar{C}$ and $Y=$ $W, \bar{W}$, i.e. conditional on consuming or not the firm's product and working or not at the firm. Notice that the corresponding conditional indirect utility functions are single-peaked. ${ }^{12}$ That does not mean at all that the unconditional indirect utility functions are single-peaked. Indeed, as price $p$ varies, the individual may get through different regimes in terms of consumption and working, and the resulting indirect utility may be double or even triple peaked. This is explained in the Appendix. Denote by

$p(C, W)=\frac{q \bar{\theta}(2 \bar{\alpha}+\bar{\theta}-\mu(\bar{\alpha}+\bar{\theta}))}{2(\bar{\alpha}+\bar{\theta})}$,

$p(C, \bar{W})=\frac{q \bar{\theta}(\bar{\theta}(1-\mu)+2 \bar{\alpha})}{2(\bar{\alpha}+\bar{\theta})}$,

$p(\bar{C}, W)=\frac{(\bar{\theta}+(2-\mu) \bar{\alpha}) q \bar{\theta}}{2(\bar{\theta}+\bar{\alpha})}$,

$p(\bar{C}, \bar{W})=\frac{q \bar{\theta}(\bar{\theta}+2 \bar{\alpha})}{2(\bar{\theta}+\bar{\alpha})} ;$

where $p(C, W)$ is the price which maximizes the utility of a shareholder who consumes the good and works in the firm, $p(\bar{C}, W)$ the price which maximizes the utility of a shareholder who only works in the company, $p(C, \bar{W})$ the price which maximizes the utility of a shareholder who only consumes the product and $p(\bar{C}, \bar{W})$ the ideal price of a pure shareholder, i.e. the profit-maximizing price. ${ }^{13}$

It is interesting, though not surprising, to see that $p(C, W)$, $p(C, \bar{W})$ and $p(\bar{C}, W)$ are decreasing in $\mu$ and tend toward the profit-maximizing price $p(\bar{C}, \bar{W})$ when $\mu$ tends toward 0 . This is of course because, as their share in the firm increases, the interests of the shareholders as consumers and/or workers become negligible with respect to their interests as owners. Indeed the profit per shareholder is increasing as $\mu$ decreases (i.e. as concentration increases). Since non-profit income is fixed (for fixed prices), profit income, whatever consumption and working decisions, becomes increasingly important for shareholders as concentration increases.

In order to obtain the main proposition below, we show in the Appendix that the ideal price of a given shareholder $(\alpha, \theta)$, the one which maximizes his/her unconditional indirect utility function (which accounts for the endogenous consumption and working decisions), is necessarily one of the four prices defined in Eqs. (3) to (6). We then determine the ideal price of a given shareholder, depending on his/her type ( $\alpha, \theta)$ and summarize the result in Fig. 2.

In Proposition 1, we first prove a stronger result than the convergence of candidate prices toward the profit-maximizing one when $\mu$ tends to zero. That is: when ownership concentration is high enough, the profit-maximizing firm's price is the ideal price of an absolute/relative majority of shareholders. In both cases (absolute or relative majority), profit-maximization obtains if the concentration of ownership is important enough and/or the incentives to work in the firm and to consume its product are low

\footnotetext{
12 More precisely, they are strictly concave with respect to $p$.

13 This is the case because the pure shareholders maximize the quantity of the numéraire which is precisely equal to their dividend, their unique revenue.
}

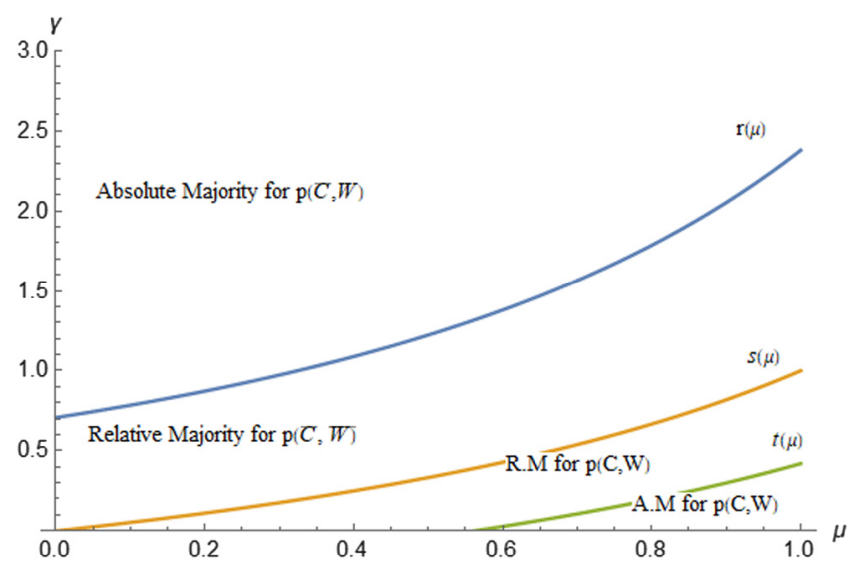

Fig. 1. Absolute/Relative majority vote in the $(\mu, \gamma)$-space.

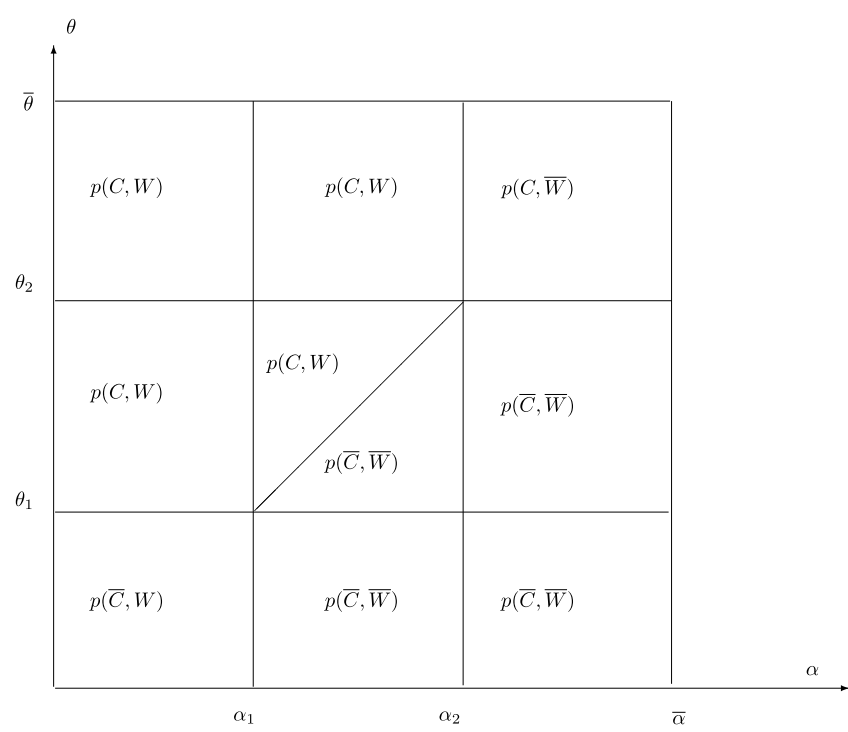

Fig. 2. The best price from the viewpoint of each individual.

enough. The condition is obviously weaker in the case of a plurality system.

Proposition 1 also provides a necessary and sufficient condition for $p(C, W)$ (a price different from the profit maximizing one) to be the ideal price of an absolute (respectively relative) majority of shareholders. When the majority is absolute, this price is the Condorcet winner.

Let now $\gamma=\frac{\bar{\alpha}}{\bar{\theta}}$, and denote by

$r(\mu)=\frac{\mu(6+\mu)+\sqrt{128+\mu^{2}(4+\mu(12+\mu))}}{8(2-\mu)}$,

$s(\mu)=\frac{\mu}{2-\mu}$,

$t(\mu)=\frac{3 \mu(2+\mu)-16+\sqrt{128+\mu^{2}(36-\mu(12+7 \mu))}}{4\left(4-\mu^{2}\right)}$.

Note that all the functions defined above are increasing, as depicted in Fig. 1.

Proposition 1. The winning price in a plurality vote is either $p(\bar{C}, \bar{W})$ or $p(C, W)$. More precisely, we have the following. 
1. The profit-maximizing firm's price $p(\bar{C}, \bar{W})$ is the ideal price of an absolute majority of shareholders if and only if

$\gamma \geq r(\mu)$

In this case, $p(\bar{C}, \bar{W})$ is the Condorcet winner.

2. The profit-maximizing firm's price $p(\bar{C}, \bar{W})$ is the ideal price of only a relative majority of shareholders if and only if

$$
s(\mu) \leq \gamma<r(\mu) .
$$

3. Price $p(C, W)$ is preferred by only a relative majority if and only if

$$
t(\mu)<\gamma \leq s(\mu) .
$$

4. Price $p(C, W)$ is preferred by an absolute majority of shareholders if and only if

$$
\gamma \leq t(\mu) \text {. }
$$

In this case, $p(C, W)$ is the Condorcet winner.

In Fig. 1 , all couples $(\mu, \gamma)$ above the curve $\gamma=r(\mu)$, i.e. satisfying Condition (10), are such that there is among the firms' owners an absolute majority (at least half) who prefers $p(\bar{C}, \bar{W})$, the profit maximizing price, to any other possible price.

Condition (11) is weaker since it ensures that only a relative majority of shareholders prefers the profit maximizing price. Both conditions mean that the incentives to work in the firm and to consume its products are limited so that a majority of the firm's owners are pure shareholders who, since they do not work nor buy the firm's products at equilibrium, seek to maximize the firm's profit. Indeed, the conditions imply a high $\bar{\alpha}$ and a low $\bar{\theta}$. As $\alpha$ measures the dis-utility of effort, a high $\bar{\alpha}$ reduces the fraction of shareholders working in the company. And as $\bar{\theta}$ measures the intensity of preference for quality, a low $\bar{\theta}$ reduces the fraction of the shareholders consuming the differentiated good. Even more interestingly, it turns out that these conditions are the more easily satisfied the smaller is $\mu$, i.e. the more concentrated is the firm's ownership. Other things equal, the more concentrated is the firm's ownership the more likely it is a profit-maximizer.

Notice that $t(\mu) \geq 0$ iff $\mu \geq \frac{1}{2}(\sqrt{17}-3) \simeq 0.561553$. Thus Condition (13), ensuring that $p(C, W)$, a price different from the profit maximizing one, be chosen by an absolute majority of owners, never holds if $\mu<\frac{1}{2}(\sqrt{17}-3) \simeq 0.561553$. Condition (13) is the less likely to be satisfied the greater is ownership concentration. This condition never holds if less than one half of the population of agents hold shares in the firm, showing the role which is played by ownership concentration in reducing the likelihood that a price other than the profit-maximizing one be chosen by the shareholders.

What is the intuition for the result that greater ownership concentration favors profit-maximization? It is useful to write a type $(\alpha, \theta)$-shareholder's utility conditional on consuming $(C)$ or not consuming $(\bar{C})$ the good and working $(W)$ or not $(\bar{W})$ as

$$
\begin{aligned}
U(X, Y)= & \frac{\pi(p(X, Y))}{\mu \bar{\alpha} \bar{\theta}}+s(X)[\theta q-p(X, Y)]+r(Y)[\omega-\alpha q]+e \\
= & \frac{\pi(p(X, Y))}{\mu \bar{\alpha} \bar{\theta}}+s(X)[\theta q-p(X, Y)] \\
& +r(Y)\left[\bar{\alpha}\left(q-\frac{p(X, Y)}{\bar{\theta}}\right)-\alpha q\right]+e,
\end{aligned}
$$

where $X=C, \bar{C}, Y=W, \bar{W}$ and $s(C)=1, s(\bar{C})=0, r(W)=1$, $r(\bar{W})=0$. The first term on the RHS is the profit per shareholder, the second one is the consumer's surplus and the third the worker's surplus. Notice that the two last terms are decreasing in the firm's price.
Obviously the number of pure shareholders is positively linked to the difference between $U(\bar{C}, \bar{W})$ and each of the three other values $U(C, W), U(C, \bar{W})$ and $U(\bar{C}, W)$. What is the effect on these differences of an increase in ownership concentration (a decrease in $\mu)$ ? We have shown that this raises the price $p(C, W)$, thus lowering both consumer's and worker's surpluses in state $(C, W)$, the price $p(C, \bar{W})$, then lowering the consumer's surplus in state $p(C, \bar{W})$ and the price $p(\bar{C}, W)$, thus lowering the worker's surplus in state $(\bar{C}, W)$. It follows that, by this channel, $U(C, W), U(C, \bar{W})$ and $U(\bar{C}, W)$ decrease while $U(\bar{C}, \bar{W})$ remains unchanged, so the differences between the latter and each of the former increase.

It remains to determine how profits per shareholder in state $(\bar{C}, \bar{W})$ change with respect to profits per shareholder in states $(C, W),(C, \bar{W})$ and $(\bar{C}, W)$. Let us consider for instance $\frac{\pi(p(\bar{C}, \bar{W}))-\pi(p(C, W))}{\mu \bar{\alpha} \bar{\theta}}$. Clearly the denominator decreases when $\mu$ decreases. Hence for the same difference in aggregate profits, the difference per shareholder increases when the fraction of the firm owned by an individual shareholder increases. As for the numerator, an increase in ownership concentration raises $p(C, W)$ while letting $p(\bar{C}, \bar{W})$ unchanged and decreases the numerator. ${ }^{14}$ It is however straightforward to show that the first effect dominates the second one: the difference in profits per shareholder increases though the difference in aggregate profits decreases.

We can safely conclude from the two combined effects on surpluses and on profits per shareholder that the equilibrium utility of a pure shareholder increases more with ownership concentration than the equilibrium utility of shareholders who are at the same time consumers and/or workers. A greater concentration of ownership raises the proportion of pure shareholders in the shareholders population.

Interestingly, ${ }^{15}$ note that, when the profit-maximizing price is selected, the number of pure shareholders (who choose not to consume the good nor to work in the firm) is strictly greater than the number of shareholders who have voted for profitmaximization. The latter equals $\mu A(p(\bar{C}, \bar{W}))$, where $(A(p(\bar{C}, \bar{W}))$ is given by Eq. (19). That is:

$$
\mu\left[\frac{\bar{\alpha} \bar{\theta}[(4-\mu) \bar{\alpha}+2 \bar{\theta}][4 \bar{\alpha}+(2-\mu) \bar{\theta}]}{16(\bar{\theta}+\bar{\alpha})^{2}}-\frac{\mu^{2} \bar{\alpha}^{2} \bar{\theta}^{2}}{8(\bar{\theta}+\bar{\alpha})^{2}}\right] ;
$$

while the former (number of pure shareholders who choose at $p(\bar{C}, \bar{W})$ not to consume nor to work), given by $\mu\left(\bar{\alpha}-\frac{\omega}{q}\right) \frac{p(\bar{C}, \bar{W})}{q}$, obtains as

$\mu \frac{\bar{\alpha} \bar{\theta}\left(\bar{\theta}+2 \bar{\alpha}^{2}\right)}{4(\bar{\theta}+\bar{\alpha})^{2}}$.

The difference (expression given by Eq. (15) minus the expression given by Eq. (14)) is given by

$\frac{\bar{\alpha} \bar{\theta}}{16(\bar{\alpha}+\bar{\theta})^{2}} \mu\left(4 \bar{\alpha}^{2}+(\mu+6) \bar{\alpha} \bar{\theta}+2 \bar{\theta}^{2}\right)$.

It is strictly positive if and only if $\mu>0$. The intuition for this result is simply that there exist some shareholders which would have preferred lower prices than the profit-maximizing one, and would have at their ideal prices consumed the product and/or worked in the firm, but who are discouraged from consuming by the high actual price and/or from working by the resulting low actual wage.

There is finally a point worth noticing. While the firm's profit is always strictly positive when the profit maximizing price is

\footnotetext{
14 Remember that the firm's profits are maximized at $p(\bar{C}, \bar{W})$.

15 We thank an anonymous referee for bringing this question to our attention.
} 
selected, this is not always the case when $p(C, W)$ is selected. Indeed in the latter case, the profit given by

$\pi(C, W)=\frac{q\left(\bar{\theta}^{2}-\mu^{2}(\bar{\theta}+\bar{\alpha})^{2}\right)}{4(\bar{\theta}+\bar{\alpha})}$,

is negative whenever $\mu>\frac{\bar{\theta}}{\bar{\theta}+\bar{\alpha}}$, i.e. when the ownership is dispersed enough. The intuition is clear. The shareholders who consume and work in the firm doubly benefit from a low price, as consumers but also as workers since a low price means a high wage (see Eq. (1)). When they hold a small enough share of the firm (dispersed ownership), this outweighs their interests as stockholders.

\section{Concluding remarks and extensions}

We introduced a simple general equilibrium model with a single firm and agents who are vertically differentiated along two dimensions, their utility for the good and their dis-utility for working. We characterized necessary and sufficient conditions under which a majority (absolute or relative) of shareholders selects the profit maximizing price and necessary and sufficient conditions under which a majority (absolute or relative) chooses a different price. The condition to have the majority choose the profit maximizing price amounts to assume that ownership concentration is great enough and/or that the average marginal utility of the good is small relative to the marginal dis-utility of working, so that the fraction of shareholders with no interest in the company as consumers or as workers is larger than one half. We have not calculated the Condorcet winner in all possible cases, i.e. in the cases when an absolute majority of shareholders do not prefer the same ideal price. However the necessary and sufficient condition for the profit-maximizing price to be a Condorcet-winner is certainly of the same type and cannot be stronger. The purpose of this note was to question the profit maximizing hypothesis while ignoring the information and manager's control issues. The results obtained in the note are sufficient for this object.

The main result of this note regards the influence of the degree of concentration of ownership. A first and immediate result is that the lower $\mu$, the more important is the weight of profits in the shareholders' utility functions. Accordingly, the three other candidate prices $p(C, W), \underline{p}(\bar{C}, W)$ and $p(C, \bar{W})$ tend toward the profit-maximizing price $p(\bar{C}, \bar{W})$ as $\mu$ tends toward 0 . In this first sense, the profit-maximization hypothesis always approximates better reality the greater is ownership concentration. But this is also true in a second stronger sense. There is a greater proportion of shareholders favoring profit-maximization over every possible alternative the greater is ownership concentration. Other things equal, there is a majority voting profit maximizing if and only if ownership concentration is above a given threshold. A contrario, we may conclude that profit-maximization may happen to fail only when (i) ownership is dispersed, (ii) an important part of the population have incentives to consume the firms' product and/or to work in the firm.

\section{Appendix}

To prove the proposition, we need two lemmas.

Denote by

$D=\left\{(\alpha, \theta) \in[0, \bar{\alpha}] \times[0, \bar{\theta}] / \frac{\theta}{\bar{\theta}}+\frac{\alpha}{\bar{\alpha}}>1\right\}$,

and by

$\bar{D}=[0, \bar{\alpha}] \times[0, \bar{\theta}] \backslash D$.

Lemma 1. The utility of each shareholder $(\alpha, \theta)$ reaches its maximal value necessarily at one of the following prices:
- $p(C, W), p(C, \bar{W})$ or $p(\bar{C}, \bar{W})$ over $D$

- $p(C, W), p(\bar{C}, W)$ or $p(\bar{C}, \bar{W})$ over $\bar{D}$.

Proof. Individual $(\alpha, \theta)$ consumes one unit of the differentiated $\operatorname{good}(C)$ if and only if $p<\theta q$.

$(\alpha, \theta)$ works $(W)$ if and only if

$\alpha<\frac{\omega}{q}$

The salary adjusts such that the labor's demand and offer equalize, so that

$\omega=\bar{\alpha}\left(q-\frac{p}{\bar{\theta}}\right)$.

The firm's profit is then the following function of its price ${ }^{16}$ :

$\pi=\bar{\alpha}\left[p\left(1+\frac{\bar{\alpha}}{\bar{\theta}}\right)-\bar{\alpha} q\right]\left[\bar{\theta}-\frac{p}{q}\right]$.

Taking into account the expression of $\omega$ as a function of $p,(\alpha, \theta)$ works if and only if $p<\bar{\theta} q\left(1-\frac{\alpha}{\bar{\alpha}}\right)$.

Comparing $\bar{\theta} q\left(1-\frac{\alpha}{\bar{\alpha}}\right)$ and $\theta q$ amounts to comparing $\frac{\theta}{\bar{\theta}}+\frac{\alpha}{\bar{\alpha}}$ with 1 .

Let us begin with the case: $(\alpha, \theta) \in D$.

As price $p$ increases, the individual $(\alpha, \theta)$ gets through three regimes:

- $(C, W)$ when $p<\bar{\theta} q\left(1-\frac{\alpha}{\bar{\alpha}}\right)$ with utility $U(C, W)=\theta q+\omega+$ $\frac{\pi}{\mu \bar{\alpha} \bar{\theta}}+e-\alpha q-p$.

- $(C, \bar{W})$ when $\bar{\theta} q\left(1-\frac{\alpha}{\bar{\alpha}}\right)<p<\theta q$ with utility $U(C, \bar{W})=$ $\theta q+\frac{\pi}{\mu \bar{\alpha} \bar{\theta}}+e-p$

- $(\bar{C}, \bar{W})$ when $p>\theta q$ with utility $U(\bar{C}, \bar{W})=\frac{\pi}{\mu \bar{\alpha} \bar{\theta}}+e$.

$p(C, W)$ maximizes the expression $U(C, W) ; p(C, \bar{W})$ maximizes the expression $U(C, \bar{W})$ and $p(\bar{C}, \bar{W})$ maximizes $U(\bar{C}, \bar{W})$. Depending on the position of these prices relative to the intervals' borders, these prices might be global maxima, just local maxima or nothing at all.

1. $p(C, W)<\bar{\theta} q\left(1-\frac{\alpha}{\bar{\alpha}}\right)$ is equivalent to

$\alpha<\frac{\bar{\alpha}(\bar{\theta}+\mu(\bar{\alpha}+\bar{\theta}))}{2(\bar{\alpha}+\bar{\theta})}(<\bar{\alpha})$.

2. $p(C, \bar{W})>\bar{\theta} q\left(1-\frac{\alpha}{\bar{\alpha}}\right)$ is equivalent to

$\alpha>\frac{(1+\mu) \bar{\alpha} \bar{\theta}}{2(\bar{\alpha}+\bar{\theta})}$.

3. $p(\bar{C}, \bar{W})>\theta q$ is equivalent to

$\theta<\frac{\bar{\theta}(2 \bar{\alpha}+\bar{\theta})}{2(\bar{\alpha}+\bar{\theta})}(<\bar{\theta})$.

4. $p(C, \bar{W})<\theta q$ is equivalent to

$$
\theta>\frac{\bar{\theta}(2 \bar{\alpha}+(1-\mu) \bar{\theta})}{2(\bar{\alpha}+\bar{\theta})}(<\bar{\theta}) .
$$

We have

$\frac{(1+\mu) \bar{\alpha} \bar{\theta}}{2(\bar{\alpha}+\bar{\theta})}<\frac{\bar{\alpha}(\bar{\theta}+\mu(\bar{\alpha}+\bar{\theta}))}{2(\bar{\alpha}+\bar{\theta})}<\bar{\alpha}$

and

$\frac{\bar{\theta}(2 \bar{\alpha}+(1-\mu) \bar{\theta})}{2(\bar{\alpha}+\bar{\theta})}<\frac{\bar{\theta}(2 \bar{\alpha}+\bar{\theta})}{2(\bar{\alpha}+\bar{\theta})}<\bar{\theta}$

$\overline{16}$ For details on the derivation of the results see Kahloul et al. (2017). 
The whole population divides into 9 zones, depending on the position of $\alpha$ relative to $\frac{(1+\mu) \bar{\alpha} \bar{\theta}}{2(\bar{\alpha}+\bar{\theta})}$ and $\frac{\bar{\alpha}(\bar{\theta}+\mu(\bar{\alpha}+\bar{\theta}))}{2(\bar{\alpha}+\bar{\theta})}$; and of $\theta$ relative to $\frac{\bar{\theta}(2 \bar{\alpha}+(1-\mu) \bar{\theta})}{2(\bar{\alpha}+\bar{\theta})}$ and $\frac{\bar{\theta}(2 \bar{\alpha}+\bar{\theta})}{2(\bar{\alpha}+\bar{\theta})}$.

Denote by $I_{1}, I_{2}$ and $I_{3}$ the 3 relevant intervals in terms of $\alpha$ in the increasing order, that is: $I_{1}=\left[0, \frac{(1+\mu) \bar{\alpha} \bar{\theta}}{2(\bar{\alpha}+\bar{\theta})}\right], I_{2}=\left[\frac{(1+\mu) \bar{\alpha} \bar{\theta}}{2(\bar{\alpha}+\bar{\theta})}\right.$, $\left.\frac{\bar{\alpha}(\bar{\theta}+\mu(\bar{\alpha}+\bar{\theta}))}{2(\bar{\alpha}+\bar{\theta})}\right]$ and $I_{3}=\left[\frac{\bar{\alpha}(\bar{\theta}+\mu(\bar{\alpha}+\bar{\theta}))}{2(\bar{\alpha}+\bar{\theta})}, \bar{\alpha}\right]$.

Denote also by $J_{1}, J_{2}$ and $J_{3}$ the 3 relevant intervals in terms of $\theta$, also in the increasing order, that is $J_{1}=\left[0, \frac{\bar{\theta}(2 \bar{\alpha}+(1-\mu) \bar{\theta})}{2(\bar{\alpha}+\bar{\theta})}\right]$, $J_{2}=\left[\frac{\bar{\theta}(2 \bar{\alpha}+(1-\mu) \bar{\theta})}{2(\bar{\alpha}+\bar{\theta})}, \frac{\bar{\theta}(2 \bar{\alpha}+\bar{\theta})}{2(\bar{\alpha}+\bar{\theta})}\right]$ and $J_{3}=\left[\frac{\bar{\theta}(2 \bar{\alpha}+\bar{\theta})}{2(\bar{\alpha}+\bar{\theta})}, \bar{\theta}\right]$.

1. $\left(I_{1} \times J_{1}\right) \cap D$ is empty.

2. On $\left(I_{2} \times J_{1}\right) \cap D$, the utility has two local maxima at $p(C, W)$ and $p(\bar{C}, \bar{W})$.

3. On $\left(I_{3} \times J_{1}\right) \cap D$, the utility reaches a global maximum at $p(\bar{C}, \bar{W})$.

4. On $\left(I_{1} \times J_{2}\right) \cap D$, the utility has two local maxima at $p(C, W)$ and $p(\bar{C}, \bar{W})$.

5. On $\left(I_{2} \times J_{2}\right) \cap D$, the utility has three local maxima at $p(C, W), p(C, \bar{W})$ and $p(\bar{C}, \bar{W})$.

6. On $\left(I_{3} \times J_{2}\right) \cap D$, the utility has two local maxima at $p(C, \bar{W})$ and $p(\bar{C}, \bar{W})$.

7. On $\left(I_{1} \times J_{3}\right) \cap D$, the utility reaches a global maximum at $p(C, W)$.

8. On $\left(I_{2} \times J_{3}\right) \cap D$, the utility has two local maxima at $p(C, \bar{W})$ and $p(C, W)$.

9. On $\left(I_{3} \times J_{3}\right) \cap D$, the utility reaches a global maximum at $p(C, \bar{W})$.

Let us consider now the case: $(\alpha, \theta) \in \bar{D}$.

As price $p$ increases, the individual $(\alpha, \theta)$ gets through three regimes:

- $(C, W)$ when $p<\theta q$ with utility $U(C, W)=\theta q+\omega+\frac{\pi}{\mu \bar{\alpha} \bar{\theta}}+$ $e-\alpha q-p$.

- $(\bar{C}, W)$ when $\theta q<p<\bar{\theta} q\left(1-\frac{\alpha}{\bar{\alpha}}\right)$, with utility $U(\bar{C}, W)=$ $\omega+\frac{\pi}{\mu \bar{\alpha} \bar{\theta}}+e-\alpha q$.

- $(\bar{C}, \bar{W})$ when $p>\bar{\theta} q\left(1-\frac{\alpha}{\bar{\alpha}}\right)$, with utility $U(\bar{C}, \bar{W})=\frac{\pi}{\mu \bar{\alpha} \bar{\theta}}+e$.

Depending on the position of prices $p(C, W), p(\bar{C}, W)$ and $p(\bar{C}, \bar{W})$ relative to the intervals' borders, these prices might be global maxima, just local maxima or nothing at all.

We have:

1. $p(C, W)<\theta q$ is equivalent to

$$
\theta>\frac{\bar{\theta}[(2-\mu) \bar{\alpha}+(1-\mu) \bar{\theta}]}{2(\bar{\alpha}+\bar{\theta})} .
$$

2. $p(\bar{C}, W)>\theta q$ is equivalent to

$$
\theta<\frac{\bar{\theta}[\bar{\theta}+(2-\mu) \bar{\alpha}]}{2(\bar{\alpha}+\bar{\theta})} .
$$

3. $p(\bar{C}, \bar{W})>\bar{\theta} q\left(1-\frac{\alpha}{\bar{\alpha}}\right)$ is equivalent to

$$
\alpha>\frac{\bar{\alpha} \bar{\theta}}{2(\bar{\alpha}+\bar{\theta})} \text {. }
$$

4. $p(\bar{C}, W)<\bar{\theta} q\left(1-\frac{\alpha}{\bar{\alpha}}\right)$ is equivalent to

$$
\alpha<\frac{\bar{\alpha}[\bar{\theta}+\mu \bar{\alpha}]}{2(\bar{\alpha}+\bar{\theta})} \text {. }
$$

We easily prove that

$\frac{\bar{\alpha} \bar{\theta}}{2(\bar{\alpha}+\bar{\theta})}<\frac{\bar{\alpha}[\bar{\theta}+\mu \bar{\alpha}]}{2(\bar{\alpha}+\bar{\theta})}<\bar{\alpha}$ and

$\frac{\bar{\theta}[(2-\mu) \bar{\alpha}+(1-\mu) \bar{\theta}]}{2(\bar{\alpha}+\bar{\theta})}<\frac{\bar{\theta}[\bar{\theta}+(2-\mu) \bar{\alpha}]}{2(\bar{\alpha}+\bar{\theta})}<\bar{\theta}$.

The whole population divides into 9 zones, depending on the position of $\alpha$ relative to $\frac{\bar{\alpha} \bar{\theta}}{2(\bar{\alpha}+\bar{\theta})}$ and $\frac{\bar{\alpha}[\bar{\theta}+\mu \bar{\alpha}]}{2(\bar{\alpha}+\bar{\theta})}$; and of $\theta$ relative to $\frac{\bar{\theta}[(2-\mu) \bar{\alpha}+(1-\mu) \bar{\theta}]}{2(\bar{\alpha}+\bar{\theta})}$ and $\frac{\bar{\theta}[\bar{\theta}+(2-\mu) \bar{\alpha}]}{2(\bar{\alpha}+\bar{\theta})}$.

Denote by $A_{1}, A_{2}$ and $A_{3}$ the 3 relevant intervals in terms of $\alpha$ in the increasing order, that is: $A_{1}=\left[0, \frac{\bar{\alpha} \bar{\theta}}{2(\bar{\alpha}+\bar{\theta})}\right], A_{2}=$ $\left[\frac{\bar{\alpha} \bar{\theta}}{2(\bar{\alpha}+\bar{\theta})}, \frac{\bar{\alpha}[\bar{\theta}+\mu \bar{\alpha}]}{2(\bar{\alpha}+\bar{\theta})}\right]$ and $A_{3}=\left[\frac{\bar{\alpha}[\bar{\theta}+\mu \bar{\alpha}]}{2(\bar{\alpha}+\bar{\theta})}, \bar{\alpha}\right]$.

Denote also by $B_{1}, B_{2}$ and $B_{3}$ the 3 relevant intervals in terms of $\theta$, also in the increasing order, that is $B_{1}=\left[0, \frac{\bar{\theta}[(2-\mu) \bar{\alpha}+(1-\mu) \bar{\theta}]}{2(\bar{\alpha}+\bar{\theta})}\right]$, $B_{2}=\left[\frac{\bar{\theta}[(2-\mu) \bar{\alpha}+(1-\mu) \bar{\theta}]}{2(\bar{\alpha}+\bar{\theta})}, \frac{\bar{\theta}[\bar{\theta}+(2-\mu) \bar{\alpha}]}{2(\bar{\alpha}+\bar{\theta})}\right]$ and $B_{3}=\left[\frac{\bar{\theta}[\bar{\theta}+(2-\mu) \bar{\alpha}]}{2(\bar{\alpha}+\bar{\theta})}, \bar{\theta}\right]$.

1. On $\left(A_{1} \times B_{1}\right) \cap \bar{D}$, as a function of $p$, the utility has a global maximum at $p(\bar{C}, W)$.

2. On $\left(A_{2} \times B_{1}\right) \cap \bar{D}$, the utility has two local maxima at $p(\bar{C}, W)$ and $p(\bar{C}, \bar{W})$.

3. On $\left(A_{3} \times B_{1}\right) \cap \bar{D}$, the utility reaches a global maximum at $p(\bar{C}, \bar{W})$.

4. On $\left(A_{1} \times B_{2}\right) \cap \bar{D}$, the utility has two local maxima at $p(C, W)$ and $p(\bar{C}, W)$.

5. On $\left(A_{2} \times B_{2}\right) \cap \bar{D}$, the utility has three local maxima at $p(C, W), p(C, \bar{W})$ and $p(\bar{C}, \bar{W})$.

6. On $\left(A_{3} \times B_{2}\right) \cap \bar{D}$, the utility has two local maxima at $p(C, W)$ and $p(\bar{C}, \bar{W})$.

7. On $\left(A_{1} \times B_{3}\right) \cap \bar{D}$, the utility reaches a global maximum at $p(C, W)$

8. On $\left(A_{2} \times B_{3}\right) \cap \bar{D}$, the utility has two local maxima at $p(\bar{C}, \bar{W})$ et $p(C, W)$.

9. $\left(A_{3} \times B_{3}\right) \cap \bar{D}$ is empty.

Lemma 2. The global maximum of the utility of Shareholder $(\alpha, \theta)$ is given by one of the prices identified in Eqs. (3), (4), (5) and (6) as shown in Fig. 2.

Proof. Utility $U(C, W)$ at $p=p(C, W)$ is given by

$U(C, W)=\frac{q(\mu \bar{\alpha}-(1-\mu) \bar{\theta})^{2}}{4 \mu(\bar{\alpha}+\bar{\theta})}+(\theta-\alpha) q$.

Utility $U(C, \bar{W})$ at $p=p(C, \bar{W})$ is given by

$U(C, \bar{W})=\theta q+\frac{\bar{\theta} q\left(\bar{\theta}(1-\mu)^{2}-4 \mu \bar{\alpha}\right)}{4 \mu(\bar{\alpha}+\bar{\theta})}$

Utility $U(\bar{C}, \bar{W})$ at $p=p(\bar{C}, \bar{W})$ is given by

$U(\bar{C}, \bar{W})=\frac{q \bar{\theta}^{2}}{4 \mu(\bar{\theta}+\bar{\alpha})}$

Utility $U(\bar{C}, W)$ at $p=p(\bar{C}, W)$ is given by

$U(\bar{C}, W)=-\alpha q+\frac{q(\bar{\theta}+\mu \bar{\alpha})\left(\bar{\theta}^{2}+(1+\mu) \bar{\alpha} \bar{\theta}+\mu \bar{\alpha}^{2}\right)}{4 \mu(\bar{\theta}+\bar{\alpha})^{2}}$

With these optimal values of indirect utilities, we have the following. $U(C, W)>U(C, \bar{W})$ iif

$$
\begin{gathered}
\alpha<\alpha_{2}=\frac{\bar{\alpha}(\mu \bar{\alpha}+2(1+\mu) \bar{\theta})}{4(\bar{\theta}+\bar{\alpha})} \\
U(\bar{C}, \bar{W})>U(C, \bar{W}) \text { iif } \\
\theta<\theta_{2}=\frac{\bar{\theta}[4 \bar{\alpha}+\bar{\theta}(2-\mu)]}{4(\bar{\theta}+\bar{\alpha})},
\end{gathered}
$$




$$
U(C, W)>U(\bar{C}, W) \text { iff }
$$

$\theta>\theta_{1}=\frac{(2-\mu) \bar{\theta}(2 \bar{\alpha}+\bar{\theta})}{4(\bar{\theta}+\bar{\alpha})}$

$$
U(\bar{C}, \bar{W})>U(\bar{C}, W) \text { iif }
$$

$\alpha>\alpha_{1}=\frac{\bar{\alpha}(\mu \bar{\alpha}+2 \bar{\theta})}{4(\bar{\theta}+\bar{\alpha})}$

$$
U(\bar{C}, \bar{W})>U(C, W) \text { iif }
$$

$\theta-\alpha<\frac{(2-\mu) \bar{\theta}^{2}-\mu \bar{\alpha}^{2}+2(1-\mu) \bar{\alpha} \bar{\theta}}{4(\bar{\theta}+\bar{\alpha})}$

We prove easily that $\alpha_{1}<\alpha_{2}<\bar{\alpha}$; $\theta_{1}<\theta_{2}<\bar{\theta}$ and that $\theta_{1}-\alpha_{1}=\theta_{2}-\alpha_{2}=\frac{(2-\mu) \bar{\theta}^{2}-\mu \bar{\alpha}^{2}+2(1-\mu) \bar{\alpha} \bar{\theta}}{4(\bar{\theta}+\bar{\alpha})}$.

Cross-checking all these inequalities leads to Fig. 2.

\section{The remaining of the proof of Proposition 1}

We calculate the relevant areas as follows.

$$
\begin{aligned}
& A(p(C, W))=\alpha_{2}\left(\bar{\theta}-\theta_{1}\right)-\frac{\left(\alpha_{2}-\alpha_{1}\right)\left(\theta_{2}-\theta_{1}\right)}{2} \\
& =\frac{\bar{\alpha} \bar{\theta}[\mu \bar{\alpha}+2(1+\mu) \bar{\theta}][2 \mu \bar{\alpha}+(2+\mu) \bar{\theta}]}{16(\bar{\theta}+\bar{\alpha})^{2}}-\frac{\mu^{2} \bar{\alpha}^{2} \bar{\theta}^{2}}{8(\bar{\theta}+\bar{\alpha})^{2}} \\
& A(p(\bar{C}, \bar{W}))=\left(\bar{\alpha}-\alpha_{1}\right) \theta_{2}-\frac{\left(\alpha_{2}-\alpha_{1}\right)\left(\theta_{2}-\theta_{1}\right)}{2} \\
& =\frac{\bar{\alpha} \bar{\theta}[(4-\mu) \bar{\alpha}+2 \bar{\theta}][4 \bar{\alpha}+(2-\mu) \bar{\theta}]}{16(\bar{\theta}+\bar{\alpha})^{2}}-\frac{\mu^{2} \bar{\alpha}^{2} \bar{\theta}^{2}}{8(\bar{\theta}+\bar{\alpha})^{2}} \\
& A(p(C, \bar{W}))=\left(\bar{\alpha}-\alpha_{2}\right)\left(\bar{\theta}-\theta_{2}\right) \\
& =\frac{(2+\mu) \bar{\alpha} \bar{\theta}^{2}[(4-\mu) \bar{\alpha}+(2-2 \mu) \bar{\theta}]}{16(\bar{\theta}+\bar{\alpha})^{2}} \\
& A(p(\bar{C}, W))=\alpha_{1} \theta_{1} \\
& =\frac{\bar{\alpha} \bar{\theta}(2-\mu)[2 \bar{\alpha}+\bar{\theta}][\mu \bar{\alpha}+2 \bar{\theta}]}{16(\bar{\theta}+\bar{\alpha})^{2}}
\end{aligned}
$$

The difference $A(p(\bar{C}, \bar{W}))-A(p(\bar{C}, W))$ which is of the same sign as $2(2-\mu) \gamma+\left(\mu^{2}-4 \mu+8\right) \gamma^{2}$, and the difference $A(p(\bar{C}, \bar{W}))-$ $A(p(C, \bar{W}))$ which is of the same sign as $2(4-\mu) \gamma^{2}+4(2-\mu) \gamma+\mu^{2}$, are always positive.

This means that $p(\bar{C}, W)$ and $p(C, \bar{W})$ are never chosen by an absolute or a relative majority.
To determine the conditions under which $p(C, W)$ is chosen by an absolute majority, we have to compare $\mu A(p(C, W))$ and $\mu \bar{\alpha} \bar{\theta} / 2$.

The difference $A(p(C, W))-\bar{\alpha} \bar{\theta} / 2$ is of the same sign as $2\left(\mu^{2}-\right.$ 4) $\gamma^{2}+\gamma\left(3 \mu^{2}+6 \mu-16\right)+2 \mu^{2}+6 \mu+4$. The result follows immediately from studying the sign of this expression.

To determine the conditions under which $p(\bar{C}, \bar{W})$ is chosen by an absolute majority, we have to compare $\mu A(p(\bar{C}, \bar{W}))$ and $\mu \bar{\alpha} \bar{\theta} / 2$. The difference $A(p(\bar{C}, \bar{W}))-\bar{\alpha} \bar{\theta} / 2$ is of the same sign as $4(2-\mu) \gamma^{2}-\mu(2+\mu) \gamma-2(2+\mu)$. The result follows immediately from studying the sign of this expression.

Now, to determine the issue of a vote by a relative majority, we have to compare $A(p(C, W))$ and $A(p(\bar{C}, \bar{W}))$. The difference $A(p(C, W))-A(p(\bar{C}, \bar{W}))$ is given by

$\frac{2 \bar{\alpha} \bar{\theta}^{3}(\mu+4)}{16(\bar{\alpha}+\bar{\theta})^{2}}\left[\gamma^{2}(\mu-2)+2 \gamma(\mu-1)+\mu\right]$,

which is of the same sign as $(\gamma+1)(2-\mu)\left(\frac{\mu}{2-\mu}-\gamma\right)$.

\section{References}

Bonnano, , 1990. General equilibrium theory with imperfect competition. J. Econ. Surv. 4, 297-328.

Codognato, G., Gabszewicz, J.J., 1991. Equilibres de Cournot-Walras dans une économie d'échange. Revue économique 42 (6), 1013-1026.

Cornwall, R., 1977. The concept of general equilibrium in a market economy with imperfectly competitive producers. Metroeconomica 29, 57-72.

Dierker, E., Grodal, B., 1999. The price normalization problem in imperfect competition and the objective of the firm. Econom. Theory 14, 257-284.

Gabszewicz, J.J., Michel, P., 1997. Oligopoly equilibria in exchange economies. In: Eaton, B. Curtis, Harris, Richard G. (Eds.), Trade, Technology and Economics. Essays in Honour of Richard G Lipsey. Edward Elgar, Cheltenham, pp. 217-240.

Gabszewicz, J.J., Vial, J.P., 1972. Oligopoly 'A La Cournot' in a general equilibrium analysis. J. Econom. Theory 4, 381-400.

Guesnerie, A., Hart, O., 1985. Welfare losses due to imperfect competition: asymptotic results for Cournot Nash equilibria with and without free entry. Internat. Econom. Rev. 26, 525-545.

Hart, , 1985. Imperfect competition in general equilibrium: an overview of recent work. In: Arrow, K.J., Honkapohja, S. (Eds.), Frontiers of Economics. Basil Blackwell, Oxford, pp. 100-149.

Kahloul, A., Lahmandi-Ayed, R., Lasram, H., Laussel, D., 2017. Vertical differentiation and labour in a general equilibrium model. J. Publ. Econ. Theory 19 (4), 860-874.

Marshall, A., 1920. [1890]. Principles of economics: An introductory volume, eighth ed. The Macmillan Press, London.

Nikaido, H., 1975. Monopolistic Competition End Effective Demand. Princeton University Press.

Persson, T., Tabellini, G.E., 2002. Political Economics: Explaining Economic Policy. The MIT Press. 